\title{
Discriminating constant from variable angular velocities in structure from motion
}

\author{
FULVIO DOMINI and CORRADO CAUDEK \\ Cognitive Technology Laboratory, AREA Science Park, Trieste, Italy \\ JESSICA TURNER \\ University of Califormia, Irvine, California \\ and
}

\author{
ALESSIA FAVRETTO \\ University of Trieste, Trieste, Italy
}

\begin{abstract}
We investigated accuracy in discriminating between constant and variable angular velocities for orthographic projections of three-dimensional rotating objects. The reported judgments of "constant" or "variable" angular velocity were only slightly influenced by the projected angular velocities, but they were greatly affected by the variations of the deformation, a first-order component of the optic flow. When viewing either a rotating ellipsoidal volume or a planar surface that accelerated and decelerated over the course of rotation, observers' tendencies to report a variable angular velocity were increased when the temporal phase of the acceleration pattern increased the range of variation of the median deformation; the tendencies were decreased when the same acceleration pattern was used to decrease the range of variation of the median deformation. These results provide evidence contrary to the hypothesis that the visual system performs a mathematically correct analysis of the optic flow.
\end{abstract}

Under certain conditions, the human observer can extract three-dimensional (3-D) information on the basis of a gradient of velocities projected onto the retina. Any changing retinal projection, however, can be the product of infinitely many combinations of different 3-D structures and motions. To obtain a unique 3-D interpretation of a changing retinal projection, it is necessary to make assumptions about the properties of the distal objects or about their motions. Several assumptions have been proposed - notably, the rigidity assumption (Ullman, 1979), smoothness of the flow field (Hildreth, 1984), fixed-axis motion (Hoffman \& Bennett, 1986), and rotation at a constant angular velocity (Hoffman \& Bennett, 1985), among others.

In the present paper, we evaluate whether the visual system's performance is consistent with a mathematically correct analysis of the stimulus information, in a situation relevant for one of the constraints proposed by the computational approach to the structure-from-motion (SFM) problem: the assumption of rotation at a constant angular velocity (Hoffman \& Bennett, 1985). If this assumption is being used by the human visual system within a mathematically correct analysis of the stimulus information, then a rigid object rotating with a constant angular velocity

The authors would like to thank J. T. Todd for valuable discussions, and M. L. Braunstein and S. Richman for helpful comments on an earlier version of this manuscript. Correspondence should be addressed to F. Domini, Department of Cognitive Sciences, School of Social Sciences, University of California, Irvine, CA 92697-5100 (e-mail: domini (a) aris.ss.uci.edu). should be perceived by observers as doing precisely thatrotating with a constant angular velocity. However, if the visual system is not able to do a mathematically correct analysis of the stimulus, a rigid object rotating with a variable angular velocity might be perceived as being a different rigid object yet rotating with a constant angular velocity, if the display is compatible with such an interpretation.

This possibility of mistaking a variable angular velocity for a constant one has been hypothesized by Domini, Caudek, and Proffitt (1997), who proposed a heuristic model predicting systematic misperceptions in the perceptual derivation of angular velocity from the optic flow. According to this model, the perception of 3-D angular velocity should be neither veridical (as would follow from a mathematically correct analysis of the stimulus information) nor biased toward being constant for an object (a possibility discussed by Liter, Braunstein, \& Hoffman, 1993). Instead, perceived 3-D angular velocity should be heuristically derived from the first-order optic flow. Domini et al. proposed that perceived angular velocity is a monotonically increasing function of the deformation (def), one of the four components into which the optic flow can be uniquely decomposed (the others being translation, rotation, and isotropic expansion or contraction; see Koenderink \& van Doorn 1975, 1976). From this hypothesis, it follows that def, rather than simulated angular velocity, should be the primary determinant of perceived 3-D angular velocity: If def is kept constant, then a constant 3-D angular velocity should be perceived; if the def variation in the optic flow is great enough, then a varying angular velocity should be perceived, regardless 


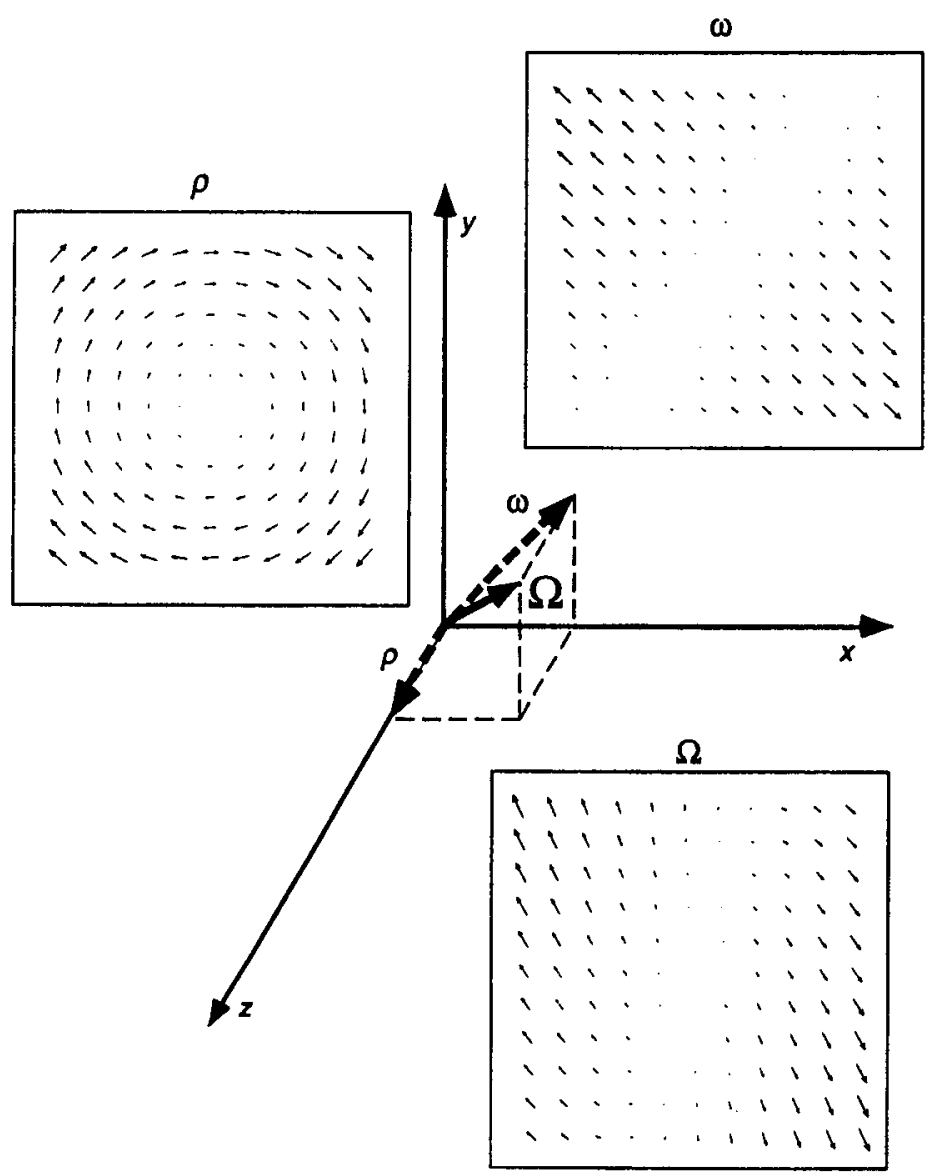

Figure 1. Decomposition of the 3-D angular velocity vector $\Omega$ into a compo-
nent $\omega$ in the $(x, y)$ plane and a component $\rho$ along the $z$-axis. In the bottom
right panel is represented the velocity field produced by the generic rotation $\Omega$.
In the left and right panels are represented the velocity fields produced by the
rotation components $\rho$ and $\omega$, respectively.

of the simulated 3-D angular velocity. Even though several sources of information have been found to influence perceived 3-D angular velocity (2-D velocity magnitude, Kaiser \& Calderone, 1991; edge transition rate, Kaiser \& Calderone, 1991; visual texture, Norman \& Todd, 1994; object size, Kaiser, 1990; deformation of contours, Cortese \& Andersen, 1991, and Norman \& Todd, 1994), the purpose of the present paper was to study the perceptual effect of the def component of the velocity field in stimulus displays in which all the other sources of information have been controlled.

\section{HEURISTIC DERIVATION OF 3-D ANGULAR VELOCITY FROM THE FIRST-ORDER OPTIC FLOW}

For the purposes of the present discussion, the relevant properties of $d e f$ can be discussed with reference to a planar patch. The orientation of a planar patch in 3-D space can be described in terms of its slant, $\sigma$ (the tangent of the angle between the line of sight or $z$-axis and the normal to the patch), and its tilt, $\tau$ (the angle between the projection of the normal to the patch onto the $x-y$ plane and the horizontal or $x$-axis). The generic angular velocity can be decomposed into two components (see Figure 1): one parallel $(\omega)$ and one orthogonal $(\rho)$ to the image plane. The global angular velocity, $\Omega$, therefore, can be expressed as

$$
\Omega=\sqrt{\omega^{2}+\rho^{2}} .
$$

It can be shown that the def component of the optic flow is equal to the product of the slant of the patch, $\sigma$, and the component of angular velocity, $\omega$, parallel to the image plane (see Domini et al., 1997):

$$
d e f=\sigma \omega .
$$

Figure 1 illustrates the relation between def and the $\sigma$ and $\omega$ components of the global velocity vector (see also Koenderink, 1986). The bottom panel shows a linear velocity field produced by the orthographic projection of a planar patch rotating about a generic axis. In this figure, the global velocity field has been decomposed into two 

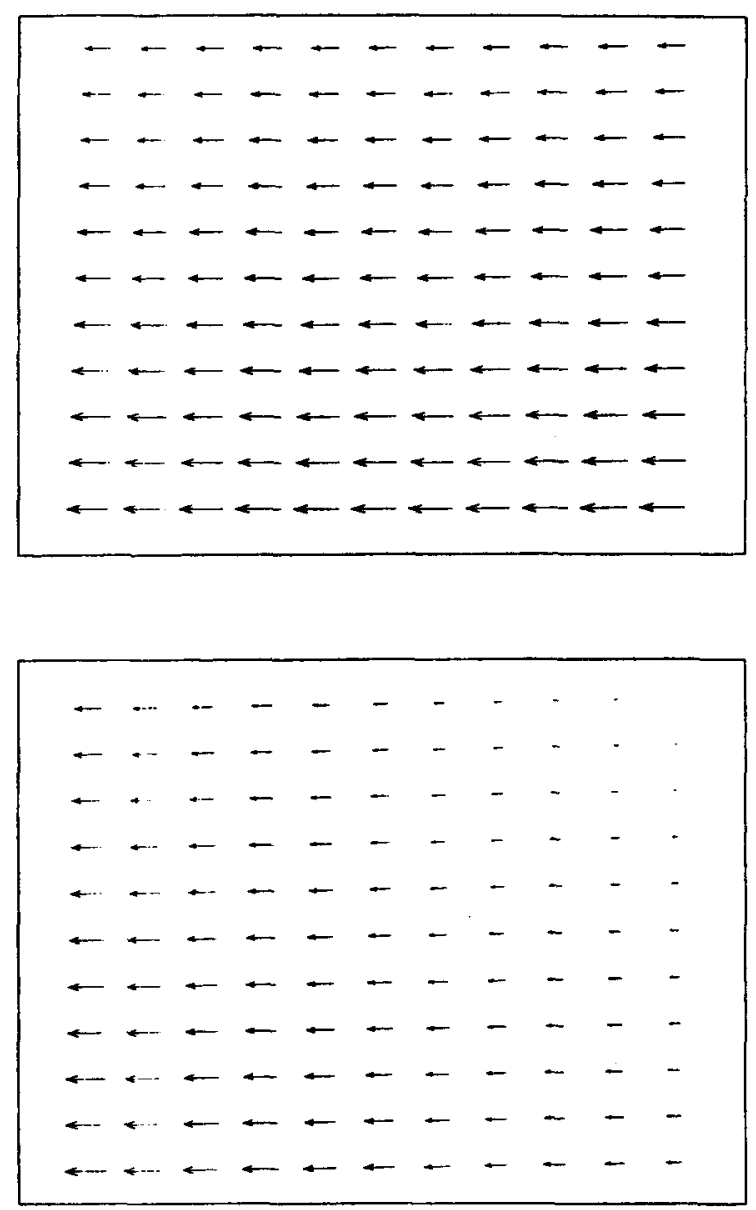

Figure 2. Velocity fields produced by the rigid rotation of a planar patch about the vertical axis. Top panel: The velocity field generated by a constant 3-D angular velocity and a slant of $20^{\circ}$. Bottom panel: The velocity field generated by a constant 3-D angular velocity and a slant of $80^{\circ}$.

components: the component representing the magnitude of rotation about an axis orthogonal to the image plane $(\rho)$ (top right panel), and the component representing the magnitude of rotation about an axis parallel to the image plane $(\omega)$ (top left panel). A rotation about an axis orthogonal to the image plane produces $2-\mathrm{D}$ rigid motion, whereas a rotation about an axis parallel to the image plane produces 2-D nonrigid motion (and parallel trajectories).

We can distinguish between rigid and nonrigid 2-D motion by considering the vectors depicted in the figure. Even though these vectors represent instantaneous velocities, they can be thought of as 2-D displacements associated with a small 3-D rotation. If the projected 2-D distance between any two points of the planar surface remains constant during the rotation, then the 2-D motion is rigid. Conversely, if this distance changes, then the 2-D motion is nonrigid. With this definition, we can think of def as a measure of the amount of 2-D nonrigidity produced by the orthographic projection of a 3-D rigid motion. As can be seen in Figure 1, def is unaffected by the magnitude of the angular velocity component $\rho$, whereas it increases with increasing $\omega$.

The relationship between def and slant is illustrated in Figure 2, which shows the velocity field produced by the orthographic projection of a planar surface rigidly rotating about the vertical axis. At the beginning of a rotation of $80^{\circ}$, the surface is slanted $20^{\circ}$ about the $x$-axis (top panel). (Note that the velocities of points closer to the vertical rotation axis, at the top, are slower than those farther from the rotation axis.) During the rotation, the slant of the surface increases. If the angular velocity is constant, then the increase of slant produces an increase of $d e f$ (see Equation 2). In Figure 2, the increase of def is revealed by the larger magnitude of 2-D nonrigidity at the end of the rotation (bottom panel) relative to the magnitude of 2-D nonrigidity at the beginning of the rotation.

It is important to realize, however, that def does not uniquely specify the two parameters by which it is defined (i.e., the slant, $\sigma$, and the rotational component parallel to the image plane, $\omega$ ). The same def, in fact, is compatible with a one-parameter family of solutions for $\sigma$ and $\omega$, which can be represented by the loci of points of the hyperbola described by Equation 2.

Since there is no evidence that the perceptual system uses the second-order temporal derivatives of the optic flow (Todd, Akerstrom, Reichel, \& Hayes, 1988; Todd \& Bressan, 1990; Todd \& Norman, 1991; see also Domini et al., 1997, and Liter, Braunstein, \& Hoffman, 1994) that are necessary to uniquely recover the projected $\omega$, we hypothesize that the perceived component of angular velocity parallel to the image plane $\left(\omega^{\prime}\right)$ is chosen heuristically as a monotonically increasing function of $\operatorname{def}$ (see Domini, Caudek, \& Gerbino, 1995; Domini et al., 1997):

$$
\omega^{\prime}=f_{1}(\text { def })
$$

The component of angular rotation about the line of sight $(\rho)$ can be correctly derived from the first-order properties of the optic flow (Hoffman, 1982). Therefore, we hypothesize that the perceived component of angular velocity orthogonal to the image plane, $\rho^{\prime}$, is at least an increasing function of the simulated component:

$$
\rho^{\prime}=f_{2}(\rho) \text {. }
$$

Finally, we hypothesize that the perceived global angular velocity is derived by combining the perceived components $\rho^{\prime}$ and $\omega^{\prime}$ as follows:

$$
\Omega^{\prime}=\sqrt{f_{1}(\text { def })^{2}+f_{2}(\rho)^{2}} .
$$

For three points, def can be computed from the 2-D coordinates of the orthogonal projection of the points and their instantaneous 2-D velocities (see Domini et al., 1997). For more than three points not lying on a planar surface, the def values for all triplets of points form a distribution. The mean def value can be very sensitive to extreme values; the median value is more representative of the central tendency of the def values. If we hypothesize that the median def value is used, then the model described by Equation 5 both accounts for findings on the percep- 
tion of 3-D angular velocity discussed below and makes several predictions that were explicitly tested in the experiments presented in this paper.

Previous researchers have found that perceived angular velocity is very close to the simulated angular velocity. Kaiser (1990) investigated the perception of 3-D angular velocity by considering the discrimination thresholds for the angular velocities of two simultaneously viewed solid cubes and other shapes. Kaiser found that observers were able to discriminate angular velocities with a competence near that for linear velocities (McKee, 1981) but that perceived velocity was biased by the number of faces revealed during rotation and by object size (i.e., smaller objects had to rotate faster than larger objects to rotate with perceptually the same velocity, and the same was true for objects with a smaller number of faces). Kaiser and Calderone (1991) extended these findings by considering rotating simulated spheres defined by either random or regularly spaced texture elements on their surfaces. Again, they found that "the motion parameter accounting for most of the variance in observers' judgments is the true angular velocity, $\omega$. Whereas extraneous spatiotemporal characteristics of the stimuli shift PSEs [points of subjective equality], these shifts are relatively minor" (p. 433).

Further results supporting the hypothesis that perceived angular velocities are a function of the simulated angular velocities are provided by Petersik (1991). In three experiments, the subjects compared the angular velocities of two rotating spheres of random dots. The results agreed with the results of Kaiser and Calderone (1991): The PSEs were close to the objective equality. Furthermore, the PSEs obtained from the direct estimates (judgments of the amount of angular rotation) of the 3-D angular velocities of the spheres agreed with the PSEs from the indirect estimates (comparing the angular velocity of two simultaneously viewed rotating spheres). In a third experiment, Petersik varied the sphere diameter and showed that, although rotation judgments are biased by mean linear velocity, they are not likely to be made solely on the basis of that information. In summary, the psychophysical data provided by these studies indicate a good sensitivity to the higher order parameter defined by $3-\mathrm{D}$ angular velocity.

Two principal findings summarize the data of Kaiser and Calderone (1991) and Petersik (1991): First, the angular velocities of two rotating spheres are correctly matched by the subjects, almost independently of the sizes of the spheres. Since the sizes are correlated with the 2-D velocities, it seems that the subjects' judgments are not influenced by the 2-D velocities. Second, the direct estimation of the angular velocity of a rotating sphere is a monotonically increasing function of the simulated angular velocity.

Both of these results can be accounted for by the model described by Equation 5 . The median of the def values for each triplet of feature points produced by clouds of dots contained within spheres having different diameters and the same angular velocity is the same. In fact, changing the size of a 3-D object isotropically leaves the slants of all the triplets of feature points unchanged. Thus, in- creasing a sphere's radius and keeping 3-D angular velocity constant have no effect on the median def, and 3-D angular velocity derived by Equation 5 is not affected by such a manipulation. This is true whether the object's optic flow results from a perspective or an orthographic projection (see the Appendix). On the other hand, increasing the simulated 3-D angular velocity of such spheres increases the median def, since it leaves the median slants of all the triplets of feature points unchanged but increases the component of angular velocity parallel to the image plane (see Equation 2). Thus, unless the axis of rotation is changed to decrease the component of angular velocity along the line of sight $\rho$, according to Equation 5, increasing the simulated 3-D angular velocity must increase the perceived 3-D angular velocity for these spheres.

There are two predictions of Equation 5 for perceived 3-D angular velocity $\Omega^{\prime}$, which we directly tested in the following experiments. First, if angular velocity $\Omega$ and the component in the image plane $\omega$ are constant, but slant $\sigma$ increases or decreases, the perceived angular velocity should increase or decrease as well. The prediction holds whether $\rho$, the component of angular velocity around the line of sight, is zero or nonzero. As long as $\rho$ is constant, the predicted $\rho^{\prime}$ is also constant, so that increasing def should increase perceived angular velocity.

Second, if the average slant $\sigma$ increases or decreases and it is possible to manipulate angular velocity $\omega$ in such a way that the product of the two is constant, and $\rho$ is constant, then perceived 3-D angular velocity should also be constant. Thus, we can predict a dissociation between simulated and perceived angular velocity: Under certain conditions, observers will see a simulated constant angular velocity as varying and, under other conditions, a simulated varying angular velocity as constant.

In the first experiment, we tested the effect of varying deformation on the perceived angular velocity of spheres and ellipsoids rotating about axes in the image plane (i.e., where $\rho$ is zero). In the second experiment, $\operatorname{def}$ and angular velocity were manipulated independently on planes rotating about axes in the image plane. In the third experiment, the axes of rotation were no longer in the image plane, and the ratios of maximum to minimum def and maximum to minimum angular velocity were controlled.

\section{EXPERIMENT 1}

In the previous research discussed above, the shapes used to study the perceived 3-D angular velocity were spheres (Kaiser \& Calderone, 1991; Petersik, 1991). Dots in a spherical volume define an isotropic structure, since the points are spread with the same probability in all directions. On the other hand, dots in the volume of an ellipsoid define an anisotropic structure, since the dots are spread with greater probability in the direction of the major axis. For points in a spherical volume, the median def is fairly constant over $180^{\circ}$ of rotation at a constant angular velocity. If the original dots are placed isotropically over the volume, subsequent rotations do not change the average slant calculated over all the triplets of dots; 


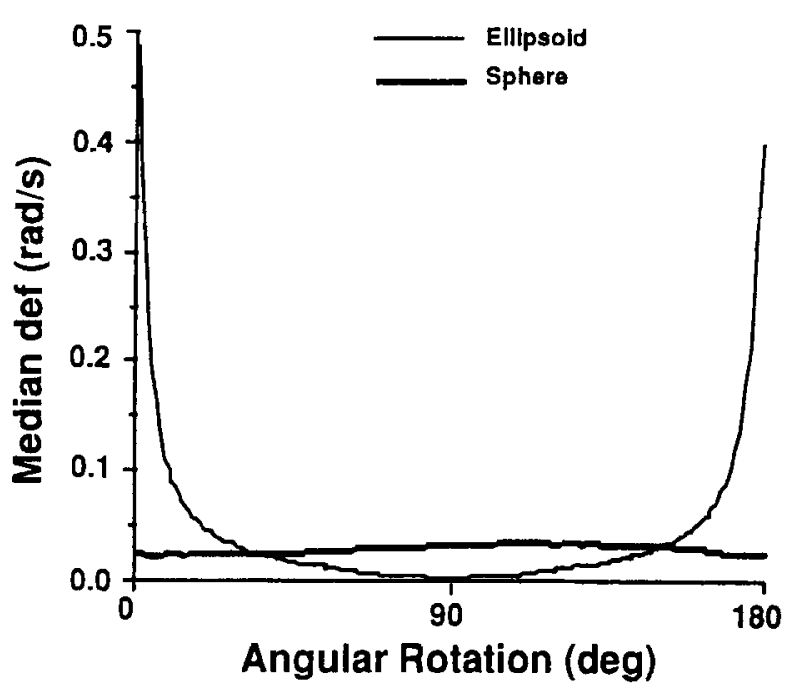

Figure 3, Median def values for all the triplets of 20 dots randomly distributed in a spherical volume and for all the triplets of $\mathbf{2 0}$ dots randomly distributed in an ellipsoidal volume, as a function of 3-D angular rotation. The ratios of the three axes of the ellipsoid to the diameter of the sphere are 3:1,0.1:1, and 1:1. The ellipsoid was originally positioned with the $3: 1$ axis along the $z$ axis ( $0^{\circ}$ of rotation), the $0.1: 1$ axis along the $x$-axis, and the 1:1 axis along the $y$-axis. In every frame transition, the two shapes rotated $1^{\circ}$ about the $y$-axis. The figure indicates that, unlike the median def of the ellipsoid, the median def of the sphere is roughly constant from frame to frame.

thus, the median def value is not greatly affected by the rotation (see Equation 2 ). This is not true for objects with anisotropic dots distributions; the distribution of slant values calculated over all triplets of dots is affected by the orientation of the object relative to the line of sight. Therefore, the median def will vary with the amount of rotation for an object with a finite number of axes of symmetry under a generic rotation, as shown for the sphere and ellipsoid in Figure 3.

By using a spherical or ellipsoidal volume in this experiment, we could show observers displays with varying or close to constant median def values. If the observers' perceptions of angular velocity were affected by the median def as hypothesized, the ellipsoidal volumes were expected to be seen as varying in angular velocity more often than the spherical volumes.

Beyond this, we could control the variation of the median $d e f$ value by accelerating and decelerating the objects during different portions of their rotations. An ellipsoid with a variable angular velocity need not have the large variation in median def that it has under a constant angular velocity. The simulated angular velocity can vary by a fixed amount while having the effect either of increasing or decreasing the median def value, depending on the phase of acceleration during the rotational cycle. When rotating at a constant angular velocity about a vertical axis in the image plane, the ellipsoid's median def values are at a maximum when its major axis is oriented along the line of sight and are at a minimum when the major axis is oriented orthogonally to the line of sight (see Figure 4).
If the ellipsoid is decelerated when its major axis is oriented along the line of sight (where the def values are at a maximum), then the def values will be decreased. Similarly, if it is accelerated when its major axis is orthogonal to the line of sight, the def values there will be increased. Over the entire rotation, the range of def variation will be reduced relative to the range of def variation in a constant angular rotation. Similarly, if the ellipsoid is accelerated when the major axis is parallel to the line of sight and decelerated $90^{\circ}$ later, the def variation is increased relative to that of a constant angular velocity.

If the simulated 3-D angular velocity is the primary influence on perceived angular velocity, then given the same amount of variation in the simulated angular velocity, the probability of perceiving a variable angular velocity should not be affected by the particular phase of the cycle in which the variation occurs. If the median def is the primary influence, however, then we would expect that accelerating the object in phase to increase the range of median def would increase reports of varying angular velocity. Similarly, accelerating the object in phase to decrease the range of median $d e f$ would decrease reports of varying angular velocity.

In a pilot experiment, we studied the effect of def on the ability to distinguish constant and variable angular velocity. We simulated a random-dot ellipsoid rotating at either a constant or a variable angular velocity $\Omega$. The constant angular velocity produced a def variation in each stimulus display as indicated in Figure 3. The varying angular velocity was computed to produce a constant def for each stimulus display. In these conditions, if the main determinant of the perceived angular velocity $\Omega^{\prime}$ were the simu-

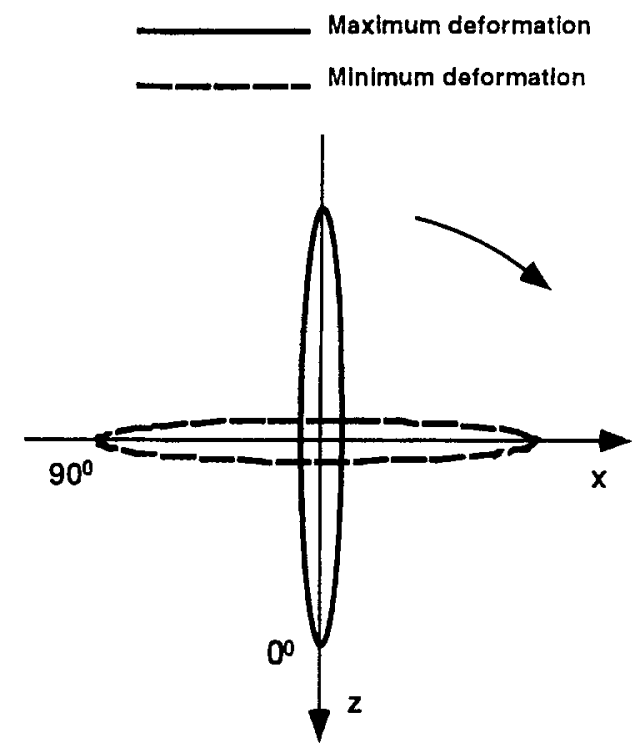

Figure 4. Schematic representation of an ellipsoidal volume in two moments of its rotation. The median def produced by the dots contained within the volume of the ellipsoid is at its maximum when the major axis of the ellipsoid is parallel to the line of sight $(z$-axis) and is at its minimum when its major axis is parallel to the $x$-axis. 


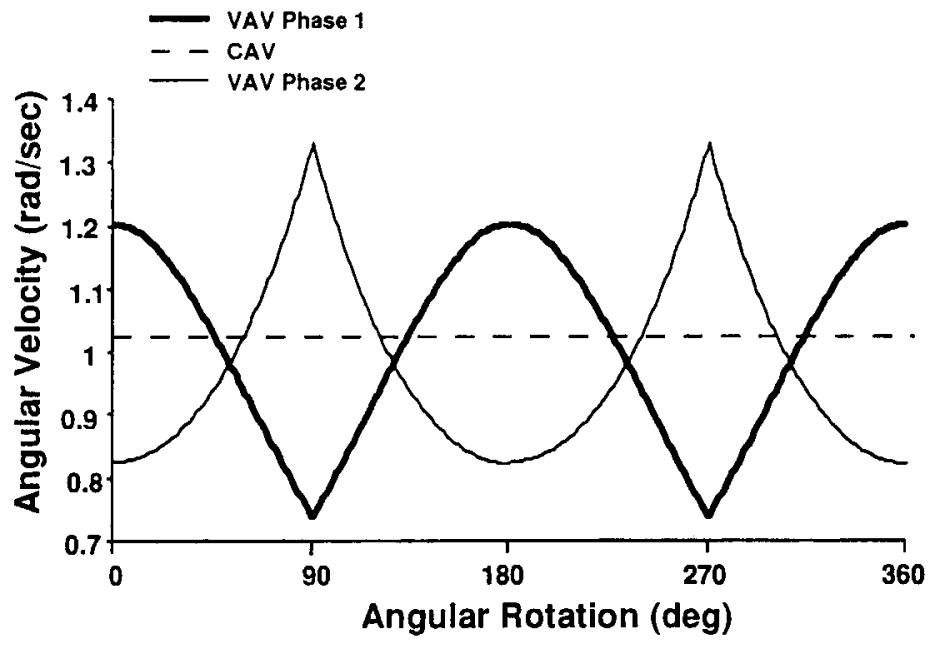

Figure 5. The 3-D angular velocities over the full rotation in each condition of Experiment 1. The sphere and ellipsoid underwent the same velocity variations. For the ellipsoid, the VAV Phase 1 modulation increased the range of def variation, whereas the VAV Phase 2 modulation decreased it.

lated angular velocity, then the judgments should be correct; if the main determinant of the perceived angular velocity were $d e f$, then the judgments should not be correct. The results of this pilot experiment provided no evidence that $d e f$ influenced perceived angular velocity, since, in general, the judgments of the observers were correct.

This failure of def to influence the judgments of perceived angular velocity was probably due to the deformation of the projected contours of the ellipsoid. This interpretation is supported by the data of Cortese and Andersen (1991), who found that the information provided by the deforming silhouettes of rotating objects is sufficient for the perceptual derivation of angular velocity. In our stimuli, then, the deformation of the 2-D contours could have overridden the effect of $d e f$.

In this first experiment, we wanted to see if $d e f$ could be made more salient than in the pilot experiment, to influence the judgments of perceived 3-D angular velocity even when the stimuli provided enough information for veridical judgments (i.e., contour deformation and texture gradients). In this experiment, we simulated randomdot ellipsoids and spheres rotating with either a constant or a varying angular velocity. There were two conditions with varying angular velocity for the ellipsoids. In one condition, the simulated angular velocity was accelerated so as to increase the range of median def relative to the constant angular velocity condition by $20 \%$ (varying angular velocity (VAV) Phase 1 condition; see Figure 5). In the second condition, the simulated angular velocity was accelerated so as to decrease the range of def relative to the constant angular velocity condition by $20 \%$ (VAV Phase 2 condition; see Figure 5). We predicted that the observers would judge the ellipsoid to be rotating with a varying angular velocity in the VAV Phase 1 condition more often than in the VAV Phase 2 condition, in accordance with the def model.
Rotating spheres were shown either rotating at a constant angular velocity or undergoing the same acceleration and deceleration patterns as the ellipsoids. Since, for a sphere, the median slant values of every triplet of points remain constant during rotation, the median $d e f$ is proportional to the simulated angular velocity (see Equation 2). Therefore, we predicted that the performance should be closer to veridical for the sphere than for the ellipsoid.

\section{Method}

Subjects. Thirteen University of Trieste undergraduates participated in this experiment. All of them were naive to the purpose of the experiment. All subjects had normal or corrected-to-normal vision. They were not paid for their participation.

Apparatus. The stimuli were presented on a high-resolution color monitor $(1,280 \times 1,024$ addressable locations), under the control of a Silicon Graphics IRIS workstation. The screen had a refresh rate of $60 \mathrm{~Hz}$ and was approximately photometrically linearized. Given the on-line calculations of the display, the actual frame rate was 58 frames $/ \mathrm{sec}$. An anti-aliasing procedure was used: For point-light locations falling on a pixel boundary, the screen luminance was proportionally adjusted in the relevant addressable locations. The graphics buffer was 8 bits deep ( 256 gray levels).

The subjects viewed the displays through a reduction screen that reduced the field of view to a circular area with a diameter $2^{\circ}$ of visual angle. The eye-to-screen distance was $1.1 \mathrm{~m}$.

Design. Two variables were studied in this experiment: (1) shape (sphere vs. ellipsoid) and (2) angular velocity variation (constant angular velocity [CAV], VAV Phase 1, and VAV Phase 2). All variables were within subjects. Each subject viewed 10 presentations of the six conditions in one block, with the order of all trials completely randomized. Twelve additional trials were presented at the beginning of each experimental session to familiarize the subjects with the stimulus displays.

Stimuli. A stimulus display consisted of 20 high-luminance dots moving on a low-luminance background. The horizontal motions of the dots simulated an orthographic projection of dots undergoing a continuous rotation in 3-D $\left(360^{\circ}\right)$ about the (vertical) $y$-axis. The dots were randomly distributed within the volume of either a sphere or an ellipsoid. The diameter of the sphere measured $2^{\circ}$ of visual 


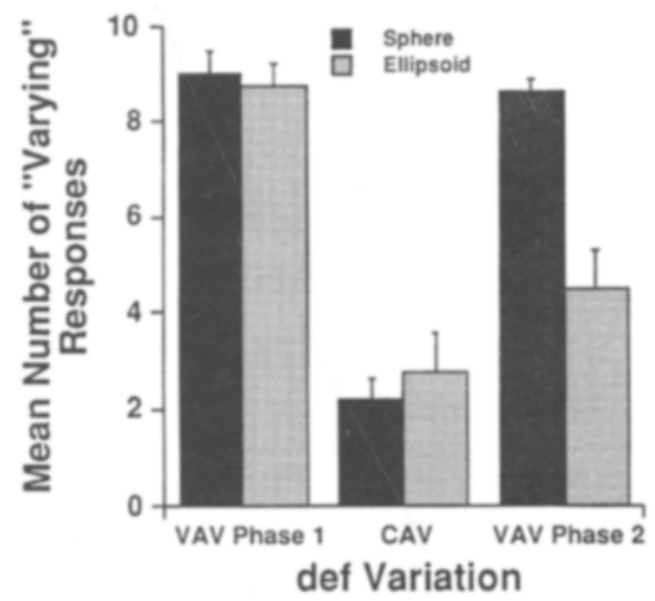

Figure 6. The mean number of trials (out of 10 possible) in which the observers reported a varying angular velocity in each condition of Experiment 1. Vertical bars represent one standard error.

angle. The ratios of the three axes of the ellipsoid to the diameter of the sphere were $3: 1,1: 1$, and $0.1: 1$. At the beginning of the rotation, the ellipsoid was oriented so that its smallest axis was parallel to the $x$-axis of the projection plane (the horizontal axis) and its major axis coincided with the $z$-axis (the line of sight). Each stimulus display was contained within a circular "window" with a diameter equal in size to the diameter of the sphere.

Both the sphere and the ellipsoid rotated with either a constant or a variable angular velocity. The range of angular velocity for both spheres and ellipsoids is shown in Figure 5. All displays simulated continuous $360^{\circ}$ rotation until terminated by the subject's response (365 frames were needed to show the full rotation, on average).

The CAV condition simulated rotation at a constant angular velocity of $1.03 \mathrm{rad} / \mathrm{sec}$. For this condition, def remained approximately constant in the case of the spheres. On the other hand, def varied for the ellipsoids as indicated in Figure 3.

The two other conditions simulated rotation at a variable angular velocity. The VAV Phase 1 and Phase 2 conditions were created by manipulating the phase of the acceleration-deceleration cycle for each stimulus display (see Figure 5). The ranges of simulated angular velocities for both conditions were identical. For the spheres, the range of variation of def in the VAV Phase 1 condition was identical to the range of variation of def in the VAV Phase 2 condition. Def was computed by calculating the median def of all triplets of points in each pair of successive frames. For the ellipsoids, the VAV Phase 1 and VAV Phase 2 conditions were created as follows.

For the VAV Phase 1 condition, when the ellipsoids were oriented with their major axis parallel to the $z$-axis, the velocity was increased (see Figure 4). This produced a larger def. When the ellipsoids were oriented with their major axis orthogonal to the $z$-axis, the velocity was decreased, producing a smaller def. This manipulation increased the range of def values in the whole rotation cycle relative to the $\mathrm{CAV}$ condition.

The VAV Phase 2 condition was produced in the opposite fashion. When the ellipsoids were oriented with their major axis parallel to the $z$-axis, then the velocity was decreased. This produced a smaller def. When the ellipsoids were oriented with their major axis orthogonal to the $z$-axis, the velocity was increased, producing a larger def. In this way, the range of def values in the whole rotation cycle was decreased relative to the CAV condition.

For the ellipsoids, in the VAV Phase 1 condition, the range of def was $20 \%$ larger than the range of def in the constant velocity con- dition. In the VAV Phase 2 condition, the range of def was $20 \%$ smaller than the range of def in the CAV condition.

Procedure. All subjects were run individually in one session. The subjects were instructed to judge whether the simulated objects appeared to rotate in 3-D with a constant angular velocity. In each trial, the subjects provided their judgments by a keypress. Viewing was monocular, and head and eye motions were not restricted. The experimental room was dark during the experiment. No restriction was placed on viewing time. No feedback was given until after the experiment was completed.

\section{Results}

The mean number of times (out of 10 repetitions) that the subjects reported a varying angular velocity for each condition is shown in Figure 6. On the average, the subjects were $76 \%$ correct, reporting a varying angular velocity in $76 \%$ of the trials in which angular velocity varied and reporting a constant angular velocity in $75 \%$ of the trials in which angular velocity was constant. The mean percent correct for all sphere conditions was $85 \%$ and for the ellipsoids was $68 \%$.

We transformed the frequency of reporting a varying angular velocity for each condition by an arcsine transformation (Winer, 1971). A 2 (shape: sphere, ellipsoid) $\times 3$ (angular velocity variation: CAV, VAV Phase 1 , VAV Phase 2) within-subjects analysis of variance (ANOVA) was run on the arcsine-transformed frequency data. Most importantly, the angular velocity variation condition had a significant effect and accounted for the most variance $\left[F(2,14)=90.89, p<.001, \omega^{2}=.61\right]$. A planned comparison between the VAV Phase 1 and VAV Phase 2 conditions on the ellipsoids alone showed that, in the VAV Phase 1 condition, the tendency to report a varying angular velocity was significantly higher $(87.5 \%)$ than in the VAV Phase 2 condition $(45.0 \%)[F(1,7)=31.12, p<.001]$. On the other hand, for the sphere, a planned comparison between the VAV Phase 1 and VAV Phase 2 conditions did not show a significant difference $[F(1,7)=2.98$, n.s. $]$.

The interaction between shape and the angular velocity variation was significant, as can be seen in Figure 6 $\left[F(2,14)=6.87, p<.01, \omega^{2}=.07\right]$. As expected, the frequency of reporting a varying angular velocity in the VAV Phase 2 condition was much greater for the sphere displays than for the ellipsoid displays. As a result, the main effect of shape was also significant $[F(1,7)=6.01, p<$ $\left..05, \omega^{2}=.03\right]$. The sphere was more frequently reported as varying in angular velocity ( $66 \%$ on the average) than was the ellipsoid (53\% on the average). Even though we expected that the frequency of reporting a varying angular velocity for the ellipsoid should be greater in the CAV condition than in the VAV Phase 2 condition, a planned comparison between these conditions did not reveal a significant difference $[F(1,7)=2.35$, n.s. $]$.

\section{Discussion}

The subjects performed quite well on the sphere displays, missing approximately $22 \%$ when the angular velocity was constant and $10 \%$ when the angular velocity was varying. This was as predicted since, for the sphere, 
the variations in $\operatorname{def}$ correlated with the variations in $3-\mathrm{D}$ angular velocity, and $\rho$ was equal to zero. The VAV Phase 1 and Phase 2 displays for the sphere were identical with respect to the variation in $\operatorname{def}$ (except for a phase shift in the temporal sequence of the stimulus display), and the tendency to report a varying angular velocity was quite similar in both conditions.

However, for the ellipsoid, only in the VAV Phase 1 condition was the tendency to report a varying angular velocity quite high. On the other hand, in the VAV Phase 2 condition, only $45 \%$ of the displays were seen correctly (i.e., with the angular velocity varying). Even though the range of angular velocity variation was the same as in the VAV Phase 1 condition, accelerating and decelerating in order to minimize def variation had the predicted effect of increasing the tendency to perceive a constant angular velocity.

When the ellipsoid rotated at a constant angular velocity, def was not constant, yet the subjects reported a constant angular velocity on approximately $73 \%$ of the trials. It seems that the changing texture density and the (partially available) contour information were able to override the changing def information and give a percept of a constant angular velocity. In Experiment 2, these characteristics were controlled, in order to further test the heuristic derivation of 3-D angular velocity described by Equation 5 .

\section{EXPERIMENT 2}

For the shapes of Experiment 1, the variation in 3-D angular velocity was accompanied by variations in the shape of the contour and in the texture density in the display. By using a planar surface whose edges extend beyond the visible window and by controlling the density of the dots on the surface, both contour and density cues can be controlled.

The predictions of the heuristic model described by Equation 5 are that angular velocity will be perceived to be constant if $d e f$ and $\rho$ are constant. If $\rho$ is constant and $d e f$ varies, the perceived angular velocity should vary. By using rotation axes that are in the image plane, $\rho$ was set equal to zero in all conditions in this experiment. Def and $\omega$, however, were varied independently of each other. Varying $\omega$ in this case is equivalent to varying the surface's 3 -D angular velocity $\Omega$ since the other component, $\rho$, is zero. Def was varied during a rotation in two ways: either by varying $\omega$ (if the slant $\sigma$ was constant) or by varying $\sigma$ (if the angular velocity component $\omega$ was constant).

It is possible that the absolute amount of def variation necessary to affect angular velocity perception is a function of the average angular velocity present. To determine any possible effect of the mean angular velocity, we manipulated the mean 3-D angular velocity of the surface by decreasing the amount of rotation between frames, while increasing the number of frames and keeping the frame rate constant. This kept the total amount of 3-D rotation constant.

While the $2-\mathrm{D}$ velocities were not explicitly manipulated as a variable in this experiment, the variation in 2-D velocity in each condition was also computed. The 2-D velocity tended to have a large variation when the 3-D angular velocity varied and tended to have a smaller variation when the 3-D angular velocity was constant. The implications of this will be discussed below.

\section{Method}

Subjects. Eighteen University of Trieste undergraduates participated in this experiment. All of them were naive to the purpose of the experiment, had normal or corrected-to-normal vision, and volunteered their time.

Apparatus. The apparatus was the same as in Experiment 1.

Design. Three variables were studied in this experiment: (1) mean angular velocity $(.044, .029$, and $.022 \mathrm{rad} / \mathrm{sec}),(2) 3-\mathrm{D}$ angular velocity variation (constant vs. variable), and (3) def variation (constant vs. variable). All variables were within subjects. Each subject viewed 10 presentations of the 12 different conditions; the 120 trials were presented in a random order. Twelve additional trials were presented at the beginning of each experimental session for practice.

Stimuli. The stimuli were 40 high-luminance dots moving on a low-luminance background. The motions of the dots simulated an orthographic projection of a planar surface oscillating in 3-D about an axis in the projection plane. Each stimulus display was contained within a circular "window" with a diameter of $2^{\circ}$ of visual angle to prevent changes in the projected contours of the simulated surfaces from being visible. The dots were randomly distributed with uniform probability density over the projection plane. Dot density was controlled by randomly deleting (or adding) dots to keep the number of dots constant in each frame of the stimulus display.

During each oscillation cycle, simulated $3-\mathrm{D}$ angular velocity and $d e f$ were kept constant or varied independently. This created four conditions: constant angular velocity/constant $\operatorname{def}$ (denoted $\mathrm{C}_{\mathrm{vel}} /$ $\left.\mathrm{C}_{\text {def }}\right)$, varying angular velocity/constant $\operatorname{def}\left(\right.$ denoted $\left.\mathrm{V}_{\text {vel }} / \mathrm{C}_{\text {def }}\right)$, constant angular velocity/varying $\operatorname{def}\left(\mathrm{C}_{\mathrm{vel}} / \mathrm{V}_{\text {def }}\right)$, and varying angular velocity/varying $\operatorname{def}\left(\mathrm{V}_{\mathrm{vel}} / \mathrm{V}_{\text {def }}\right)$. These were created by changing the orientation of the plane relative to the axis of rotation across conditions and by varying or maintaining the angular velocity within a condition. Figure 7 shows the orientation of the planar surfaces relative to the axis of rotation in each experimental condition. The tilt of the axis of rotation (and, therefore, of the planar surface) was determined randomly on each trial from $0^{\circ}$ to $360^{\circ}$.

The initial slant $\sigma$ of the surface was always 8 [i.e., $\tan \left(82.9^{\circ}\right)$ ]. When the axis of rotation is almost perpendicular to the surface, with an initial slant close to $\tan \left(90^{\circ}\right)$, a small rotation induces very little slant change. Thus, by keeping the angular velocity constant in such a condition and with a small rotation angle, we can produce a nearly constant $d e f$. Conversely, if the angular velocity varies, then the def will also vary. On the other hand, when the axis of rotation is almost parallel to the surface, with an initial slant close to $\tan \left(90^{\circ}\right)$, a small rotation induces a very large slant change. Thus, if the 3-D angular velocity is constant in such a condition, the def will still vary; but the def can also be kept constant if the 3-D angular velocity varies in such a way that the product of $\omega$ and $\sigma$ is kept constant.

In the $\mathrm{C}_{\text {vel }} / \mathrm{C}_{\text {def }}$ condition (Figure $7 \mathrm{a}$ ), the axis of rotation was almost perpendicular to the planar surface. In this way, a $6^{\circ}$ rotation produced a negligible change of the slant of the surface. Therefore, def was nearly constant since the 3-D angular velocity was constant (see Equation 2). The def varied over the course of the display by $0.3 \%, 0.7 \%$, and $0.5 \%$ in the slowest, medium, and fastest angular velocity conditions, respectively. The projected $2-\mathrm{D}$ velocity of each dot was also nearly constant during the time course of a stimulus display, varying by $0.5 \%, 0.3 \%$, and $0.5 \%$ in the three angular velocity conditions.

In the $\mathrm{V}_{\mathrm{vel}} / \mathrm{C}_{\text {def }}$ condition (Figure $7 \mathrm{~b}$ ), the axis of rotation was contained in the planar surface. A $5.6^{\circ}$ rotation produced a large change of the slant of the surface, but we compensated for the slant variation with a variation of the $3-\mathrm{D}$ angular velocity in order to keep 


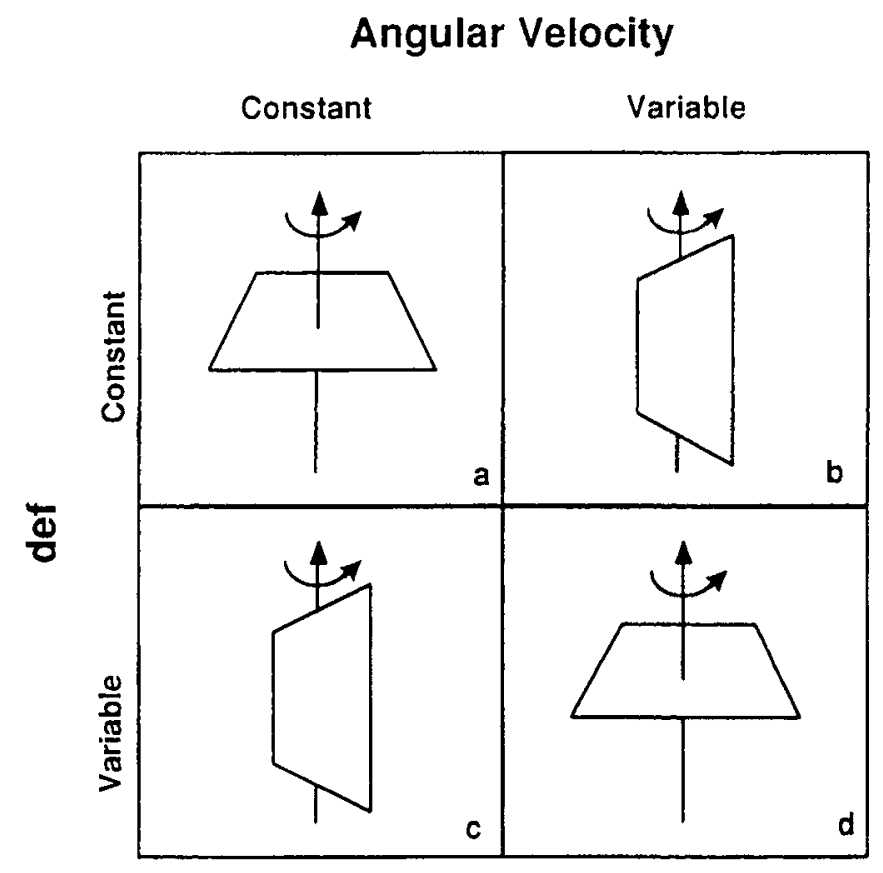

Figure 7. Orientation of the simulated planar surfaces relative to the axis of rotation in each condition of Experiment 2.

the product of the two constant. Therefore, def was constant. The projected 2-D velocity of each dot varied during the time course of a stimulus display by $494 \%, 499 \%$, and $494 \%$ in the three angular velocity conditions. The $3-\mathrm{D}$ angular velocity varied by $472 \%, 478 \%$, and $483 \%$ in the same conditions.

In the $\mathrm{C}_{\mathrm{vel}} / \mathrm{V}_{\text {def }}$ condition (Figure $7 \mathrm{c}$ ), the axis of rotation again was contained in the planar surface. In this way, a $6^{\circ}$ rotation produced a large change of the slant of the surface. Therefore, def was variable since the slant was variable. The def values varied by $622 \%$, $628 \%$, and $630 \%$ in the slowest, medium, and fastest angular velocity conditions, respectively. The projected 2-D velocity of each dot, however, was very nearly constant, never varying more than $0.9 \%$.

In the $\mathrm{V}_{\text {vel }} / \mathrm{V}_{\text {def }}$ condition (Figure $7 \mathrm{~d}$ ), the axis of rotation again was almost perpendicular to the planar surface. In this way, a $5.8^{\circ}$ rotation produced a negligible change of the slant of the surface. But since the 3-D angular velocity was variable, def was also variable. The projected 2-D velocity of each dot was also variable in this condition. The $3-\mathrm{D}$ angular velocity varied by $667 \%, 710 \%$, and $721 \%$, the def values varied by $664 \%, 704 \%$, and $718 \%$, and the $2-\mathrm{D}$ velocity varied by $587 \%, 646 \%$, and $684 \%$ in the slowest, medium, and fastest $3-\mathrm{D}$ angular velocity conditions, respectively.

In the variable angular velocity conditions, the total amount of rotation was not the same as in the constant angular velocity conditions because of the necessary manipulations to control def. In Figures 8 , 9 , and 10 are shown the 3-D angular velocity, def, and 2-D velocity for each stimulus type for each frame transition in the $0.044 \mathrm{rad} / \mathrm{sec}$ condition. The 2-D velocity plot indicates the ratio between the 2-D velocity of a dot in a frame transition and its minimum $2-D$ velocity in a frame transition across the whole stimulus sequence. We used this measure since the absolute magnitude of 2-D velocity is different for each dot of a display, but this ratio is the same for all dots in each frame transition. Since the simulated angle of rotation was very small in all conditions, when the 3-D angular velocity was constant, the 2-D projected velocity was nearly constant for each dot over the course of a display. Conversely, when the $3-\mathrm{D}$ angular velocity was variable, the $2-D$ projected velocity for each dot was also variable over the course of a display.
Each stimulus display had 40,60 , or 80 frames. Varying the number of frames while keeping the magnitude of rotation constant varied the average angular velocity for a display: $0.044,0.029$, and $0.022 \mathrm{rad} / \mathrm{sec}$ in the $40-, 60-$, and 80 -frame conditions, respectively.

Procedure. The procedure and instructions were the same as in Experiment 1.

\section{Results}

The mean number of times (out of 10 repetitions) that the subjects reported a varying angular velocity in each condition is shown in Figure 11. Overall, the mean percent correct across conditions was $61.7 \%$.

A 3 (mean angular velocity: $0.044,0.029,0.022 \mathrm{rad} /$ sec) $\times 2$ (def: constant, varying) $\times 2$ (angular velocity variation: constant, varying) within-subjects ANOVA was run on the arcsine-transformed frequency data. The effect of $d e f$ was significant and accounted for the highest proportion of variance $\left[F(1,17)=122.25, p<.001, \omega^{2}=.64\right]$. When def varied, the frequency of reporting a varying angular velocity was significantly higher $(76.3 \%)$ than when def was constant $(20.8 \%)$.

The effect of 3-D angular velocity was also significant, though it accounted for a negligible portion of the variance $\left[F(1,17)=19.19, p<.001, \omega^{2}=.02\right]$. The frequency of reporting a varying angular velocity was higher when angular velocity did vary $(53 \%)$ than when angular velocity was constant $(44 \%)$. The effect of the mean angular velocity was not significant $[F(2,34)<1.0]$.

The interaction between $d e f$ and velocity was significant $\left[F(1,17)=8.29, p<.01, \omega^{2}=.02\right]$. As can be seen in Figure 11, the effect of velocity was greater when def was constant; when def was varying, the subjects nearly always reported a varying angular velocity, whether or 


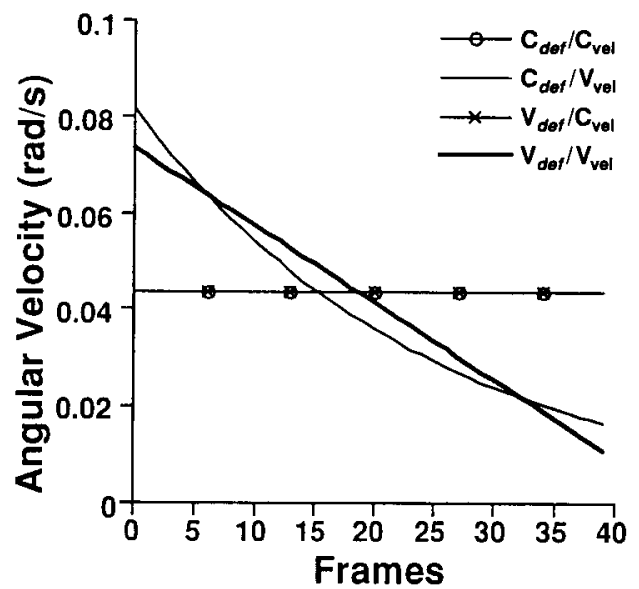

Figure 8. The 3-D angular velocity for each condition of the $0.044 \mathrm{rad} / \mathrm{sec}$ stimulus sequence of Experiment 2.

not the velocity was actually varying. The interaction between def and the mean angular velocity was also significant, though it accounted for a negligible portion of the variance $\left[F(2,34)=5.03, p<.05, \omega^{2}=.004\right]$. As mean angular velocity decreased, the difference between the effects of constant and varying def became more pronounced: At $0.044 \mathrm{rad} / \mathrm{sec}$, in the constant def condition, the mean frequency of reporting varying angular velocity was $25 \%$, and in the variable def condition, the mean frequency was $73 \%$. At $0.022 \mathrm{rad} / \mathrm{sec}$, the mean frequencies were $18 \%$ and $78 \%$, respectively.

\section{Discussion}

The results of Experiment 2 tend to support the predictions of Equation 5: Under certain conditions, the observers reported a varying angular velocity when the angular velocity $\Omega$ was constant and, under other conditions, when $\Omega$ varied. When def was constant, the observers were more likely to report a varying angular velocity correctly, but they were unable to distinguish constant from varying angular velocity when def was varying. When def varied, the observers reported a varying angular velocity on approximately $76 \%$ of the trials, though the angular velocity was varying on only half of the trials. Thus the observers tended to perceive a varying angular velocity when def varied, but not necessarily when angular velocity varied.

Similarly, the reports of varying angular velocity were not primarily determined by the $2-\mathrm{D}$ velocities: The $2-\mathrm{D}$ velocities of each dot were nearly constant over the course of a display when 3-D angular velocity was constant, and they varied when $3-D$ angular velocity varied. It is possible that when def was constant, either the variation in 2-D velocity or the variation in 3-D velocity allowed the observers to report a varying angular velocity more correctly. The effect was small, however, and accounted for only $2 \%$ of the total variance.

In conclusion, in Experiment 2, the angular velocity and the def information were decoupled, and the observers' reports of perceived variable angular velocity were predicted for the most part by def. An effect of varying the 3-
D angular velocity was found when $d e f$ was constant, but it was very small relative to the effect of $d e f$. These results were obtained for rotations about a rotation axis in the image plane. The heuristic derivation of 3-D angular velocity described by Equation 5 was formulated to take into account generic rotation axes. Experiment 3 tested the model in the more generic case.

\section{EXPERIMENT 3}

In the heuristic derivation of 3-D angular velocity described by Equation 5, perceived 3-D angular velocity is a function both of def and of $\rho$, the component of image velocity around the line of sight. In Experiments 1 and 2, only def was varied, as $\rho$ was kept constant at zero. In Experiment 3 , in one condition, $\rho$ was constant but nonzero, and, in another condition, it varied over the course of a display. If $d e f$ is held constant while $\rho$ is varied, a varying angular velocity should still be perceived.

Although Experiments 1 and 2 demonstrated that varying def can cause a varying angular velocity to be perceived, the amount of def variation was not systematically manipulated. If the perceived angular velocity is a monotonically increasing function of $d e f$ as assumed in the model, then increasing the def variation should increase the tendency to report a varying angular velocity. Similarly, increasing the variation in $\rho$ should increase the tendency to report a varying angular velocity. To test this prediction, in Experiment 3, the ratio of maximum to minimum def within a display could be one of three values $\rho$ was held constant, or the ratio of maximum to minimum $\rho$ within a display could be one of three values and $\operatorname{def}$ was held constant.

\section{Method}

Subjects. Six University of Trieste undergraduates participated in this experiment. All of them were naive to the purpose of the experiment, had normal or corrected-to-normal vision, and volunteered their time.

Apparatus. The apparatus was the same as in Experiment 1.

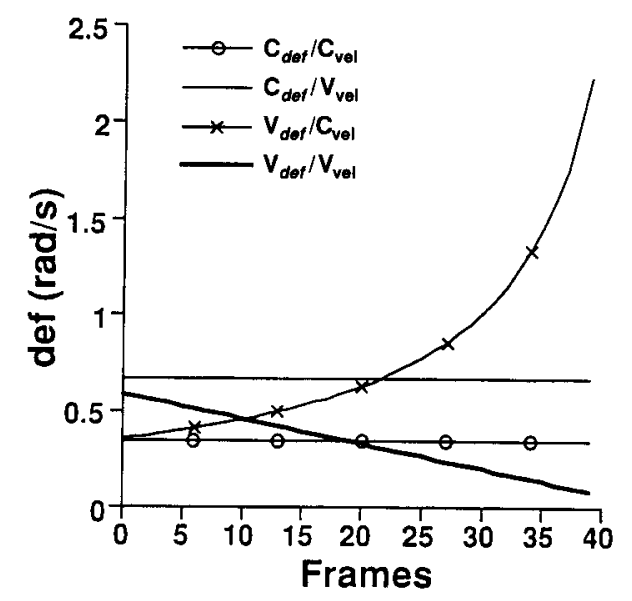

Figure 9. The def values for each condition of the $0.044 \mathrm{rad} / \mathrm{sec}$ stimulus sequence of Experiment 2. 


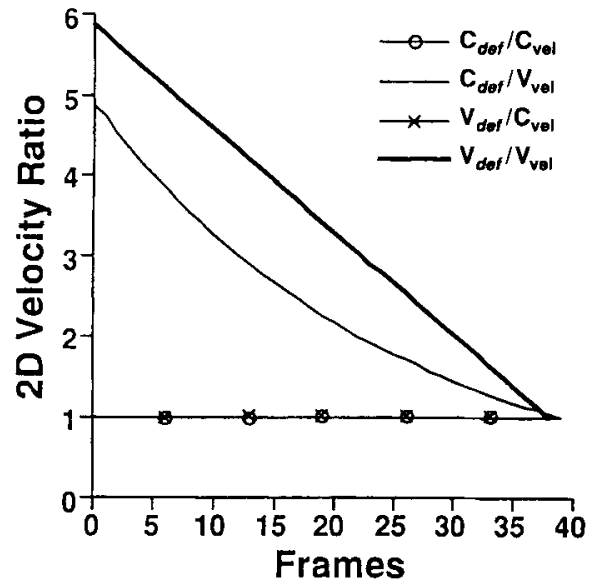

Figure 10. Ratio between the 2-D velocity of a point in each frame transition and its minimum 2-D velocity over the course of a display for each condition of the $0.044 \mathrm{rad} / \mathrm{sec}$ stimulus sequence of Experiment 2.

Design. We used two combinations of angular velocity and $d e f$, and three possible ratios between the maximum and minimum values of either 3-D angular velocity or def in a display. The two combinations of angular velocity and def were constant angular velocity/variable def $\left(\mathrm{C}_{\mathrm{vel}} / \mathrm{V}_{d e f}\right)$ and variable angular velocity/constant $\operatorname{def}\left(\mathrm{V}_{\mathrm{vel}} / \mathrm{C}_{d e f}\right)$.

If 3-D angular velocity remained constant in a display, the ratios between the maximum and minimum def could be 3,5 , or 7 . In the same way, if def remained constant, the ratios between the maximum and minimum 3-D angular velocity could be 3, 5, or 7. Each subject viewed 80 presentations in random order of the six conditions. Twelve additional trials were presented at the beginning of each experimental session for practice.

Stimuli. The stimuli were 40 high-luminance dots moving on a low-luminance background. The motions of the dots simulated an orthographic projection of a planar surface undergoing 3-D oscillation. Twenty-five possible axes of rotations were chosen from 34 generated using the same procedure as Braunstein, Hoffman, and Pollick (1990): 34 points were chosen evenly spaced on the surface of a sphere, and the axes of rotation were defined by connecting each of these points to the center of the sphere. Of the 34 axes generated this way, 9 were almost in the image plane (their slants were larger than $81^{\circ}$ ), and they were discarded. The slant of the remaining axes ranged from $45^{\circ}$ to $81^{\circ}$, and their tilt varied between $0^{\circ}$ and $180^{\circ}$.

Each stimulus display simulated a rotation about an axis contained in the simulated surface. The dots were randomly distributed with uniform probability density over the projection plane (not evenly distributed over the simulated surfaces). Dot density was manipulated as before to keep the number of dots constant in each frame of the stimulus display. Each stimulus display was contained within a circular "window" with a diameter of $2^{\circ}$ of visual angle.

Two variables were manipulated: angular velocity and def. For each trial, they could either remain constant or vary. Two stimulus conditions were defined: constant angular velocity/variable def(denoted $\mathrm{C}_{\mathrm{vel}} / \mathrm{V}_{d e f}$ ) and variable angular velocity/constant def (denoted $\mathrm{V}_{\text {vel }} / \mathrm{C}_{\text {def }}$ ).

In the $\mathrm{C}_{\mathrm{vel}} / \mathrm{V}_{d e f}$ condition, on each trial, one axis of rotation was chosen randomly from the previously defined set of axes. The planar surface was oriented parallel to the axis and with an initial slant equal to $1\left[\tan \left(45^{\circ}\right)\right]$. Each stimulus display was made up of 20 frames. The angle of rotation for each frame transition was computed using an iterative procedure so that the angular velocity was constant but the def of the last frame transition was 3,5 , or 7 times larger than the def of the first frame transition. After the frame tran- sition in which def was maximum, the screen was blackened for $200 \mathrm{msec}$. The display was repeated until the subjects provided their judgments. Angular velocity took on the values of $0.569,0.628$, and $0.648 \mathrm{rad} / \mathrm{sec}$ for the simulated def ratios of 3,5, and 7, respectively.

In the $\mathrm{V}_{\mathrm{vel}} / \mathrm{C}_{\text {def }}$ condition, the orientation of the axis of rotation on each trial was determined by randomly choosing one axis from the previously defined set. In each trial, the planar surface was rotated around the axis to have an initial slant equal to $1\left[\tan \left(45^{\circ}\right)\right]$. To obtain a constant value of def, the increment in the angle of rotation for each frame transition was the following:

$$
\Delta \alpha=\frac{\operatorname{def} \Delta t}{\sigma_{\text {surface }} \cos \left(\sigma_{\mathrm{axis}}\right)}
$$

where $\sigma_{\mathrm{axis}}$ is the slant of the axis of rotation, $\Delta t$ is the temporal duration of a frame transition, and $\sigma_{\text {surface }}$ is the slant of the simulated surface. In each stimulus display, the slant of the simulated surfaces increased monotonically during the rotation. In the last frame transition, $\Delta \alpha$ took on a value that was 3,5 , or 7 times larger than the $\Delta \alpha$ of the first frame transition. This procedure produced a different number of frame transitions in different stimulus displays depending on the orientation of the axis of rotation. The ratios between the maximum and minimum simulated 3-D angular velocities took on the values of 3,5 , or 7 . Consequently, the ratios between the maximum and minimum values of $\rho$ took on the same values. After the frame transition in which the simulated 3-D velocity was maximum, the screen was blackened for $200 \mathrm{msec}$. The display was repeated until the subjects provided their judgments. In each display, def took on the values of $0.8 \mathrm{rad} / \mathrm{sec}$ for the simulated 3-D angular velocity ratios of 3,5 , and 7 .

The average magnitude of def for each frame transition over 240 stimuli for the ratios between maximum and minimum def of 3,5, and 7 in the $\mathrm{C}_{\mathrm{vel}} / \mathrm{V}_{d e f}$ condition is shown in Figure 12. The average magnitude of $\rho$ for each frame transition over 240 stimuli for the ratios between maximum and minimum $\rho$ of 3,5 , and 7 in the $V_{v e l} / C_{d e f}$ condition is shown in Figure 13.

Procedure. The procedure and instructions were the same as in Experiment 1, except that the trials were broken up into a total of four sessions of equal length.

\section{Results}

The mean number of times (out of 80 ) that the subjects reported a varying angular velocity in each condition

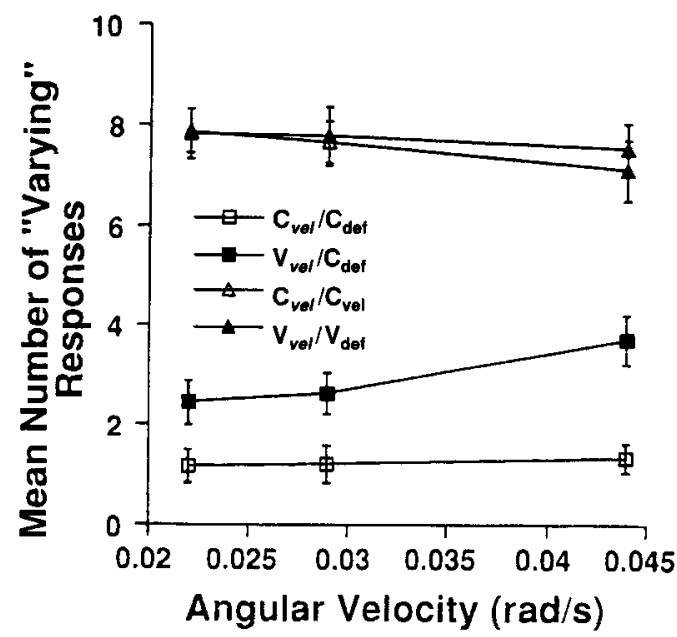

Figure 11. The mean number of trials (out of 10 possible) in which the observers reported a varying angular velocity in each condition of Experiment 2. Vertical bars represent one standard error. 


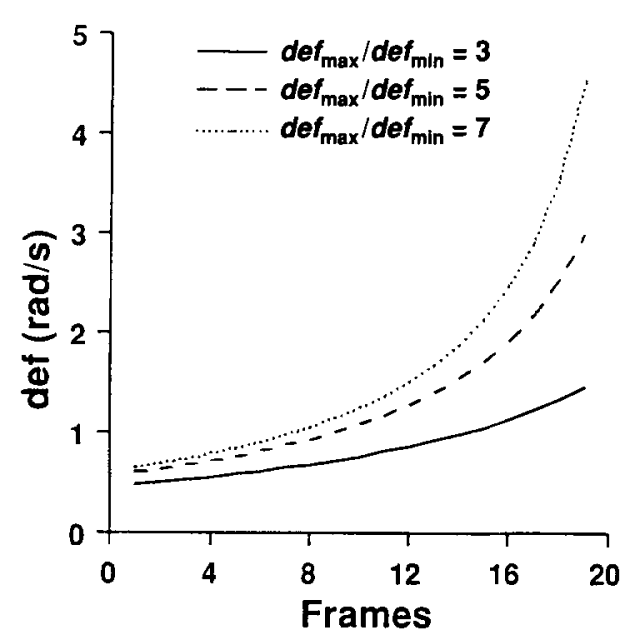

Figure 12. The def values for each ratio between maximum and minimum def in the constant angular velocity/variable $d e f$ condition of Experiment 3, over a full display cycle.

is shown in Figure 14. On the average, the subjects were $33 \%$ correct (significantly less than chance ${ }^{1}$ ), reporting a varying angular velocity on $65 \%$ of the trials in which angular velocity was constant and only on $31 \%$ of the trials in which angular velocity varied.

The effect of maximum to minimum def ratio was examined by performing a within-subjects ANOVA on the arcsine-transformed frequency data in the $\mathrm{C}_{\mathrm{vel}} / \mathrm{V}_{\text {def }}$ condition $\left[F(2,10)=83.96, p<.05, \omega^{2}=.89\right]$. Planned comparisons revealed significant differences between the smallest ratio condition and the next larger ratio condition $[F(1,5)=64.64, p<.05]$ and between the two largest ratio conditions $[F(1,5)=17.26, p<.05]$.

The effect of maximum to minimum velocity ratio was examined by performing a within-subjects ANOVA on the $\mathrm{V}_{\mathrm{ve}} / \mathrm{C}_{\text {def }}$ condition. The effect of velocity ratio was not significant $[F(2,10)=1.83$, n.s. $]$.

\section{Discussion}

The results confirm the findings of Experiments 1 and 2 , in that perceiving a varying $3-D$ angular velocity depends more on the variation in def than on whether the simulated $3-D$ angular velocity is actually varying. The observers' reports of a varying angular velocity when def varied were in a ratio of 2 to 1 with their reports of such a percept when def was constant but angular velocity varied. Thus, even when the axis of rotation is not in the image plane, but is more generically positioned, observers' perceptions of a constant or varying 3-D angular velocity depend on whether def is constant or varying. Moreover, we found that the increase of both the ratios of maximum to minimum def and the ratios of maximum to minimum $\rho$ increased the tendency of reporting a varying angular velocity, even though the effect of the variation in $\rho$ did not reach significance.

\section{GENERAL DISCUSSION}

The heuristic model described by Equation 5 predicts that the perception of 3-D angular velocity will not be veridical. Specifically, perceived 3-D angular velocity from optic flow will be an increasing function of the def component of the optic flow and the component of angular velocity around the line of sight. The predictions of this model were supported by the experimental results. In Experiment 1, increasing the range of def variation increased the tendency to report a varying angular velocity, even though the actual range of angular velocity variation was unchanged. In Experiment 2, the tendency to report a varying angular velocity was much less when def was constant than when def varied. When def varied, the tendency to report a varying angular velocity was very strong and was essentially unaffected by whether the simulated 3-D angular velocity varied or not. In Experiment 3 , increasing the range of def variation while holding angular velocity constant increased the tendency to report a varying angular velocity.

The model also predicts that a variation in $\rho$ will lead to variation in the perceived 3-D angular velocity. In Experiment 3 , the variation in $\rho$ produced by varying the 3-D angular velocity around an axis not in the image plane did affect the tendency to report a varying angular velocity. This effect was not as large as the effect of def even though the ratio of maximum to minimum $\rho$ when $\rho$ varied was the same as the ratio of $d e f$ variation when def varied. Possibly the visual system might be more sensitive to def than to $\rho$, however, and this warrants further research.

There was a small effect of the simulated 3-D angular velocity found in Experiment 2: When def was constant, varying the 3-D angular velocity did increase the tendency to report a perceived varying 3-D angular velocity.

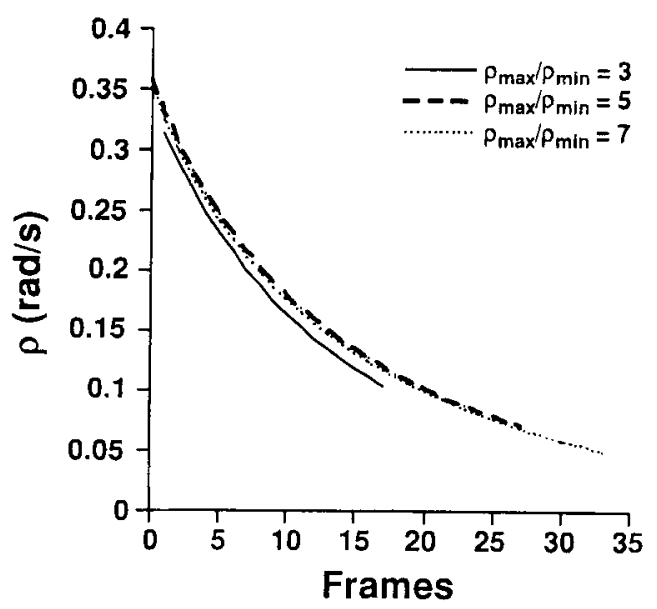

Figure 13. The $\rho$ values for each ratio between maximum and minimum 3-D angular velocity in the variable angular velocity/constant def condition of Experiment 3, over a full display cycle. 


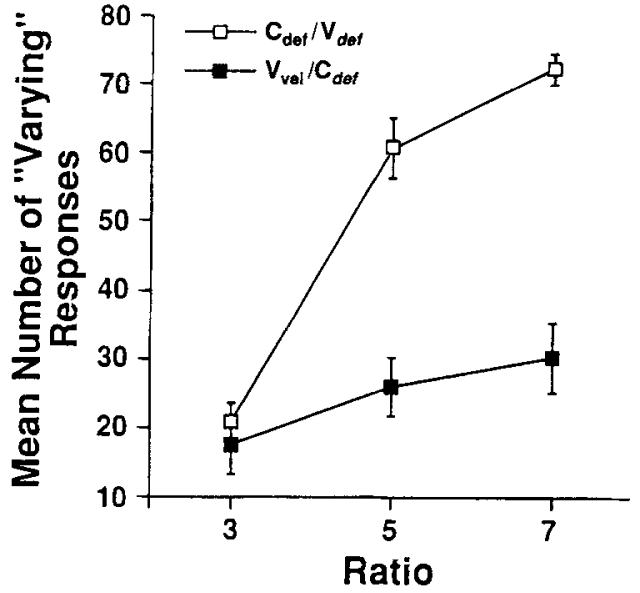

Figure 14. The mean number of trials (out of 80 possible) in which the observers reported a varying angular velocity in each condition of Experiment 3. Vertical bars represent one standard error.

On the average, however, a constant angular velocity was reported on more than $60 \%$ of the trials in which angular velocity varied while def was constant, and a variable angular velocity was reported on more than $75 \%$ of the trials in which angular velocity was constant while def varied. Within the present stimulus parameters, this argues for a primary effect of def on 3-D perceived angular velocity, rather than the simulated 3-D angular velocity as has been claimed previously (Kaiser \& Calderone, 1991; Petersik, 1991).

The tendency to report a varying 3-D angular velocity did not seem to be affected by variations in the 2-D velocities of the dots in the display. In Experiment 2, the 2-D velocity varied with the simulated angular velocity, but the variation in def had the greatest effect on whether the $3-D$ perceived angular velocity varied. Even though the ratio of maximum to minimum 2-D velocity of a dot over the display could be as high as 5.8 , when def was constant, 3-D angular velocity tended to be perceived as constant.

Def has been shown to affect several aspects of perceived structure from optic flow. The influence of def on the perception of rigidity in SFM displays has been studied by Domini et al. (1997). Moreover, Caudek and Domini (1998) studied the influence of def on the perception of the orientation of the axis of rotation. In both studies, they found that, in general, the perceptual judgments were not influenced by the simulated 3-D stimulus properties but depended mainly on the manipulation of def. The findings of these studies parallel the findings here, in that the ability to detect a varying 3-D angular velocity can depend on the variability of the median def.

The heuristic model of Equation 5 has been tested in these experiments using stimuli with only optic flow information about the angular velocity. Other sources of information about the angular velocity can exist: In particular, the results of Experiment 1 show that the effects of $d e f$ can be overcome in part by texture and boundary information, which are in themselves sources of informa- tion about the 3-D angular velocity (Cortese \& Andersen, 1991). The extent to which these and other factors and the def information interact is an issue for future research.

In summary, perceived 3-D angular velocity is not veridical, as predicted by Domini et al. (1997): 3-D angular velocity can be perceived to vary or to be constant somewhat independently of the simulated angular velocity, if the def and $\rho$ variation is large or small, respectively. This argues against the notion that the human visual system uses an assumption of rotation at a constant angular velocity to recover structure within a mathematically correct analysis of the stimulus information, and it argues for a heuristic analysis based on the first-order optic flow.

\section{REFERENCES}

Braunstein, M. L., Hoffman, D. D., \& Pollick, F. E. (1990). Discriminating rigid from nonrigid motion: Minimum points and views. Perception \& Psychophysics, 47, 205-214.

Caudek, C., \& Domini, F. (1998). Perceived orientation of axis of rotation in structure-from-motion. Journal of Experimental Psychology: Human Perception \& Performance, 24, 609-621.

Cortese, J. M., \& Andersen, G. J. (1991). Recovery of 3-D shape from deforming contours. Perception \& Psychophysics, 49, 315-327.

Domini, F, CAudek, C., \& Gerbino, W. (1995). Perception of surface attitude in structure from motion displays. Investigative Ophthalmology \& Visual Science, 36, 1694.

Domini, F., Caudek, C., \& Proffitt, D. R. (1997). Misperceptions of angular velocities influence the perception of rigidity in the kinetic depth effect. Journal of Experimental Psychology: Human Perception \& Performance, 23, $1111-1129$.

Hildreth, E. (1984). The measurement of visual motion. Cambridge, MA: MIT Press.

HoFFMAN, D. D. (1982). Inferring local surface orientation from motion fields. Journal of the Optical Society of America, 72, 888-892.

Hoffman, D. D., \& BENNETT, B. (1985). Inferring the relative 3-D positions of two moving points. Journal of the Optical Society of America $A, 2,350-353$.

Hoffman, D. D., \& BennetT, B. (1986). The computation of structure from fixed axis motion: Rigid structures. Biological Cybernetics, 54, $71-83$.

KaISER, M. K. (1990). Angular velocity discrimination. Perception \& Psychophysics, 47, 149-156.

Kaiser, M. K., \& Calderone, J. B. (1991). Factors influencing perceived angular velocity. Perception \& Psychophysics, 50, 428-434.

Koenderink, J. J. (1986). Optic flow. Vision Research, 26, 161-179.

KOENDERINK, J. J., \& VAN DOORN, A. J. (1975). Invariant properties of the motion parallax field due to the movement of rigid bodies relative to an observer. Optica Acta, 22, 773-791.

KoEnderink, J. J., \& VAN DoORN, A. J. (1976). Local structure of movement parallax of the plane. Journal of the Optical Society of America $A, 66,717-723$.

Liter, J. C., Braunstein, M. L., \& Hoffman, D. D. (1993). Inferring structure from motion in 2 -view and multi-view displays. Perception, 22, 1441-1465.

Liter, J. C., Braunstein, M. L., \& Hoffman, D. D. (1994). Detection of one versus two objects in structure from motion. Journal of the Optical Society of America A, 11, 3162-3166.

Marascuilo, L. A. (1970). Extensions of the significance test for oneparameter signal detection hypotheses. Psychometrika, 35, 237-243.

MCKEE, S. P. (1981). A local mechanism for differential velocity detection. Vision Research, 21, 491-500.

Norman, J. F., \& ToDD, J. T. (1994). Perception of rigid motion in depth from the optical deformations of shadows and occlusion boundaries. Journal of Experimental Psychology: Human Perception \& Performance, 20, 344-356.

Petersik, J. T. (1991). Perception of three-dimensional angular rotation. Perception \& Psychophysics, 50, 465-474.

Todd, J. T.. Akerstrom, R. A., Reichel, F. D., \& Hayes, W. (1988). Ap- 
parent rotation in three-dimensional space: Effects of temporal, spatial, and structural factors. Perception \& Psychophysics, 43, 179-188.

TODD, J. T., \& BressaN, P. (1990). The perception of 3-dimensional affine structure from minimal apparent motion sequences. Perception \& Psychophysics, 48, 419-430.

ToDD, J. T., \& Norman, J. F. (1991). The visual perception of smoothly curved surfaces from minimal apparent motion sequences. Perception \& Psychophysics, 50, 509-523.

UlLman, S. (1979). The interpretation of visual motion. Cambridge, MA: MIT Press.

WINER, B. J. (1971). Statistical principles in experimental design (2nd ed.). New York: McGraw-Hill.

\section{NOTE}

1. The experimental data were analyzed by means of a signal detection paradigm, considering the "varying angular velocity" trials as signal trials. A $d^{\prime}$ measure was computed for each observer. The significance of $d^{\prime}$ was calculated by using Marascuilo's (1970) one-signal significance test. The results of this analysis revealed that all six $d^{\prime} \mathrm{s}$ were significantly less than zero.

\section{APPENDIX \\ Influence of Size and Angular Velocity on Median def of Random Dot Spheres}

Stimuli similar to those of Kaiser and Calderone (1991) and Petersik (1991) were simulated under a perspective projection to de- termine their median def. Kaiser and Calderone and Petersik simulated the perspective projection of rotating random-dot spheres. For Kaiser and Calderone, the standard sphere had a diameter of $4.5^{\circ}$ of visual angle, was made up of 400 points, and was rotated at $2.5^{\circ}$ /frame. For Petersik, the standard sphere had a diameter of $11.2^{\circ}$ of visual angle, was made up of 50 dots, and was rotated at $3 \%$ frame. For Kaiser and Calderone and for Petersik, the dots were positioned either inside the spheres or on their surfaces.

To verify that median def is affected by angular velocity and not by the size of the sphere, we ran a computer simulation on a set of nine spheres made up of 50 randomly placed dots. The dots were positioned within the volume of the spheres. The simulated diameters were equal to $5^{\circ}, 10^{\circ}$, or $15^{\circ}$ of visual angle. For rotations of $2^{\circ}, 4^{\circ}$, or $6^{\circ}$, we calculated the median def of all the triplets of dots. The results of this simulation clearly indicate that median def increases with the increase of the angle of rotation (see Figure A1). The size of the sphere also slightly influenced median def, but this effect was negligible relative to the effect of the angle of rotation.

A second simulation was run to verify whether the same pattern of results can also be obtained for orthographic projections. The stimulus parameters were identical to those of the former simulation. The results were very similar to those obtained under a perspective projection: The size of the sphere did not have as much of an effect on median def as did angular velocity (see Figure A1).

\section{Perspective Projection}

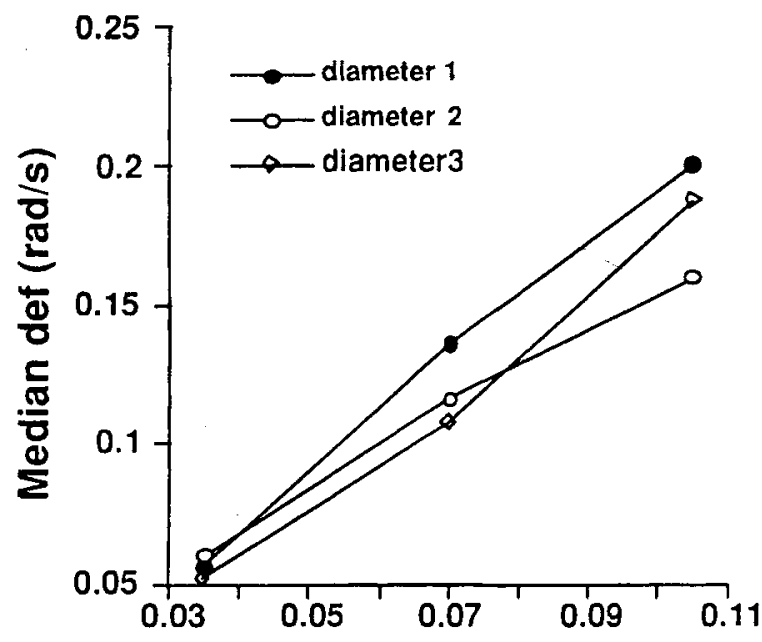

\section{Orthographic Projection}

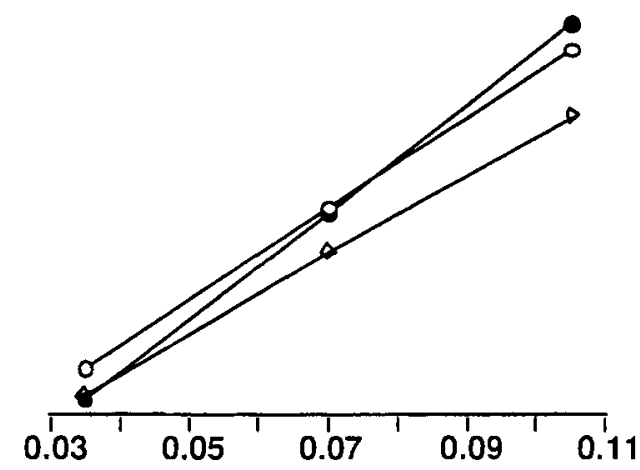

Angular Velocity ( $\mathrm{rad} / \mathrm{s})$

Figure A1. Median def as a function of 3-D angular velocity for spheres with different diameters. Perspective projection (left panel) and orthographic projection (right panel). 